\title{
A case of acute onset headache and visual loss
}

\author{
Deepa Ragesh Panikkath MD
}

\begin{abstract}
Granulomatosis with polyangiitis (GPA) is a multisystem autoimmune disorder. Typically, GPA presents with upper airway, pulmonary, and renal symptoms; an initial presentation limited to central system involvement (CNS) is rare. This case report describes a 54-year-old woman who presented with persistent daily headaches and a visual defect. Investigation revealed meningeal inflammation and optic neuritis demonstrated by magnetic resonance imaging and positive serine proteinase-3 ANCA serology diagnostic of GPA. She had a dramatic clinical response to corticosteroids. This case highlights a rare initial presentation of GPA with CNS manifestations.
\end{abstract}

Keywords: Granulomatosis with polyangiitis (GPA), pachymeningitis, anti-PR3 cANCA

\section{INTRODUCTION}

Granulomatosis with polyangiitis (GPA) is a rare idiopathic autoimmune disease. Necrotizing granulomatous inflammation and vasculitis in small and medium sized blood vessels are its hallmark features. ${ }^{1}$ In GPA, the pattern of autoantibody staining is cytoplasmic (c-ANCA), due to the presence of antibodies against proteinase 3 , a constituent of the azurophilic granules of the neutrophil. ${ }^{2}$ Apart from classically involving the lungs, upper airways, and kidneys, involvement of the nervous system has been described in $20-50 \%$ of patients. Peripheral neuropathy is more commonly seen than central nervous system manifestations. ${ }^{3}$

\section{CASE}

A 54-year-old Caucasian woman presented with a right-sided headache and right-sided facial pain of 2 months' duration. She described daily right-sided, throbbing headaches which were not associated with nausea, vomiting, or any prodromal symptoms. She

\section{Corresponding author: Deepa Panikkath}

Contact Information: deeparag@gmail.com

DOI: 10.12746/swrccc.v7i27.525 had no relief with over the counter analgesics. Her symptoms progressively got worse followed by the development of diminished vision in her right eye. This initially affected her central visual field and progressed to involve her peripheral vision. She did not have any accompanying systemic symptoms of fever, night sweats, or weight loss. Her past medical history was significant for type 2 diabetes mellitus and hypertension. She was an ex-smoker with a 60 pack-year history and had quit 6 months prior to presentation. She had a history of methamphetamine abuse in the past.

On physical examination, the patient was alert, cooperative, and in no acute distress. Her vital signs were within normal limits. Systemic examination revealed normal lung, cardiovascular, and abdominal examinations. She had tenderness over the right temporal region. Examination of the nervous system showed significant findings involving the right cranial nerves II and VI. She had defective perception to light and afferent pupillary defect along with loss of abduction in the right eye. Other cranial nerves examined were normal. She had normal motor and sensory examination with symmetric $2+$ deep tendon reflexes and no accompanying cerebellar or meningeal signs. Her gait was normal. Fundus examination showed mild blurring of the margins of the right optic disc. 
Her complete blood count revealed a hemoglobin of $14.8 \mathrm{~g} / \mathrm{dL}$, total white cell count of $9850 / \mu \mathrm{l}$, and a platelet count of $408,000 / \mu \mathrm{l}$. The erythrocyte sedimentation rate was elevated at $85 \mathrm{~mm} / \mathrm{hr}$ (normal range 0-30 $\mathrm{mm} / \mathrm{hr}$ in women over 50 ). C-reactive protein was $7.3 \mathrm{mg} / \mathrm{L}$. Blood chemistry levels were within normal limits. Urinalysis was normal with no proteinuria or casts. Autoimmune workup was done due to the presence of elevated inflammatory markers. Antinuclear antibody and anti dsDNA were negative. Serum C3 and $\mathrm{C} 4$ levels were low. She had a positive rheumatoid factor of $32 \mathrm{IU} / \mathrm{ml}$ (normal $<15$ ) and positive proteinase 3 antinuclear cytoplasmic antibody (PR-3 ANCA) at $>8 \mathrm{Al}$ (normal $<1$ ). Anti-myeloperoxidase antibodies were negative. Her chest $\mathrm{x}$-ray was within normal limits. Computed tomography (CT) of the head and sinuses demonstrated bilateral maxillary, ethmoid, and sphenoid sinus opacification without bony erosions. CT angiography of the head and neck was normal. Cerebral magnetic resonance imaging with gadolinium (MRI) showed asymmetric enlargement of the infraorbital and canalicular portion of the right optic nerve suggestive of optic neuritis (Figure 1). No intraparenchymal abnormalities or evidence of venous sinus thrombosis were detected. She had significant meningeal thickening and enhancement in the frontal region that represented pachymeningitis (Figure 2). The patient refused to undergo a lumbar puncture for cerebral spinal fluid studies.

Based on the above workup with positive anti PR3 ANCA levels and cranial inflammation, she was diagnosed with GPA and started on high dose intravenous corticosteroids (1-gram methylprednisolone daily for 5 days) followed with oral prednisone of $100 \mathrm{mg}$ a day on a slow taper schedule. She had a dramatic improvement in her vision and headaches by day 3 . She was subsequently discharged with advice to follow up for outpatient immunosuppressant therapy, but information from follow-up clinic visits is not available.

\section{Discussion}

This case report describes a patient who presented with pachymeningitis and cranial nerve involvement as the initial manifestation of GPA. This case of limited GPA differs from the classic multisystem GPA. Limited

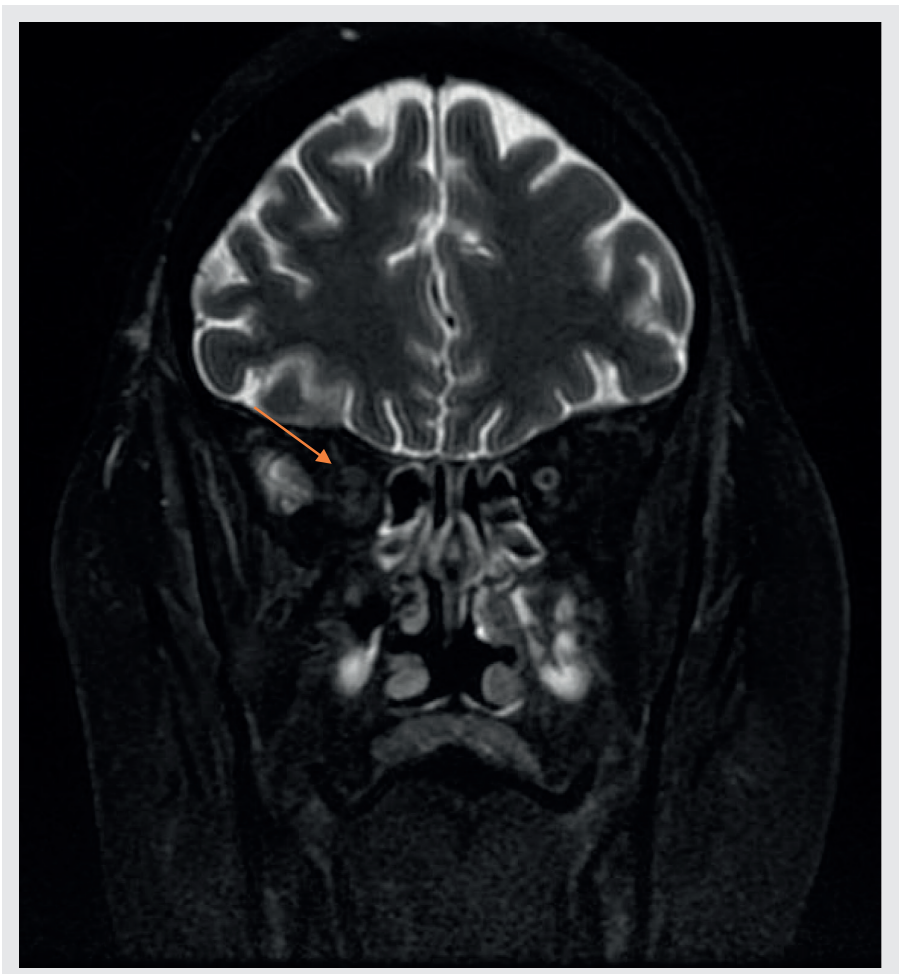

Figure 1. Cerebral magnetic resonance imaging with gadolinium (MRI) showing asymmetric enlargement of the infraorbital and canalicular portion of the right optic nerve suggestive of optic neuritis.

forms of GPA have limited organ involvement, usually respond to less toxic immunosuppressant regimes, and have a better prognosis. A quarter of these cases have negative ANCA serology which makes them difficult to diagnose. ${ }^{4,5}$ Central nervous system involvement occurs in $2-8 \%$ of the patients with GPA, and dysfunction of cranial nerves, commonly the second, third, sixth, and seventh nerves, often occurs in these cases. Meningeal involvement is extremely rare. In two large series, meningeal involvement was observed in zero of the $158(0 \%)$ patients and in 2 of the $324(0.6 \%)$ patients with GPA. ${ }^{6,7}$ Three major mechanisms are postulated to cause CNS disease and include contiguous invasion of granuloma from extracranial sites, remote intracranial granulomas, and vasculitis. ${ }^{8}$ Other CNS manifestations include cerebrovascular events, seizures, and 


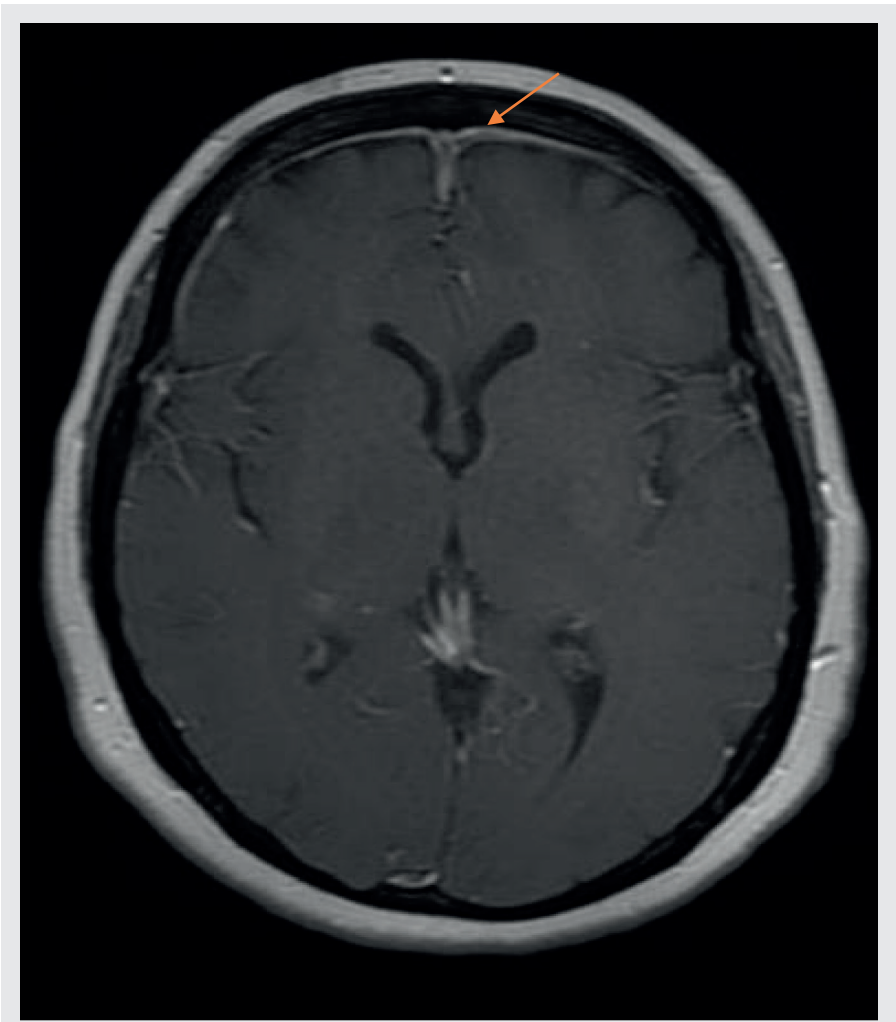

Figure 2. MRI showing significant meningeal thickening and enhancement in the frontal region representing pachymeningitis.

reversible posterior leukoencephalopathy syndrome. PR3-ANCA is reported to be strongly associated with the disease in over $90 \%$ of GPA patients with active disease..$^{9}$ This patient had a dramatic improvement in her symptoms with treatment with glucocorticoids which along with immunosuppressants remain the main treatment modality of this disease.

\section{Conclusion}

Granulomatosis with polyangiitis is an important differential diagnosis in patients with CNS manifestations associated with elevated inflammatory markers. Rapid diagnostic work-up and therapeutic intervention with immunosuppressant drugs are necessary to prevent or reduce potential permanent damage in patients with CNS manifestations.

Article citation: Panikkath DR. A case of acute onset headache and visual loss. The Southwest Respiratory and Critical Care Chronicles 2019;7(27):55-57

From: The Department of Internal Medicine at Texas Tech University Health Sciences Center in Lubbock, Texas

Submitted: 9/28/2018

Accepted: $12 / 10 / 2018$

Reviewers: James Tarbox MD, John Pixley MD

Conflicts of interest: none

This work is licensed under a Creative Commons Attribution-ShareAlike 4.0 International License.

\section{REFERENCES}

1. Falk RJ, Gross WL, Guillevin L, et al. Granulomatosis with polyangiitis (Wegener's): an alternative name for Wegener's granulomatosis. Arthritis Rheum 2011;63:863-864.

2. Comarmond C, Cacoub P. Granulomatosis with polyangiitis (Wegener): clinical aspects and treatment. Autoimmun Rev 2014;11:1121-1125.

3. Holle JU, Gross WL. Neurological involvement in Wegener's granulomatosis. Curr Opin Rheumatol 2011;1:7-11.

4. Carrington CB, Liebow AA. Limited form of angiitis and granulomatosis of Wegener's granulomatosis of Wegener's type. Am J Med 1966;41:497-527.

5. Stone JH. Wegener's Granulomatosis Etanercept Trial Research Group Limited versus severe Wegener's granulomatosis: baseline data on patients in the Wegener's granulomatosis etanercept trial. Arthritis Rheum. 2003;48:2299-2309.

6. Hoffman GS, Kerr GS, Leavitt RY, et al. Wegener granulomatosis: an analysis of 158 patients. Ann Intern Med 1992;116: 488-498.

7. Nishino H, Rubino FA, DeRemee RA, et al. Neurological involvement in Wegener's granulomatosis: an analysis of 324 consecutive patients at the Mayo Clinic. Ann Neurol 1993;33:4-9.

8. Murphy JM, Gomez-Anson B, Gillard JH, et al. Wegener granulomatosis: MR imaging findings in brain and meninges. Radiology 1999;213:794-799.

9. Finkielman JD, Lee AS, Hummel AM, et al. ANCA are detectable in nearly all patients with active severe Wegener's Granulomatosis. Am J Med. 2007;120:643.e9-14. 